\title{
Aquaporin 3 promotes prostate cancer cell motility and invasion via extracellular signal-regulated kinase 1/2-mediated matrix metalloproteinase-3 secretion
}

\author{
JIE CHEN* ${ }^{*}$ ZHIJUN WANG* , DANFENG XU, YUSHAN LIU and YI GAO \\ Department of Urology, Changzheng Hospital, Second Military Medical University, \\ Shanghai 200003, P.R. China
}

Received February 16, 2014; Accepted November 3, 2014

DOI: $10.3892 / \mathrm{mmr} .2014 .3097$

\begin{abstract}
Aquaporins (AQPs) are known to be important in cancer progression. The present study used a cDNA microarray to demonstrate that AQP3, a member of the AQP family, was overexpressed in prostate cancer cells. In order to determine the role of AQP3 in prostate cancer, AQP3 expression was silenced by transfection with small interfering RNA, following which wound healing and invasion assays were conducted in DU-145 and PC-3 cells. Notably, the results showed that silencing of AQP3 suppressed prostate cancer cell motility and invasion. The possible mechanisms underlying the AQP3-enhanced motility and invasion of prostate cancer cells was also investigated. Knockdown of AQP3 expression was shown to reduce extracellular signal-regulated kinase $1 / 2$ (ERK1/2) activation in DU-145 and PC-3 cells. Blocking the ERK pathway inhibited AQP3-mediated motility and invasion of these cells. Finally, the results demonstrated that AQP3 upregulated matrix metalloproteinase-3 (MMP-3) expression and secretion in prostate cancer cells via activation of the ERK pathway. In conclusion, the present study suggests that AQP3 induces ERK1/2 activation, thereby increasing MMP-3 expression and secretion, which in turn promotes prostate cancer cell motility and invasion. Thus, AQP3 may be considered to be a potential therapeutic target for prostate cancer.
\end{abstract}

Correspondence to: Dr Yi Gao, Department of Urology, Changzheng Hospital, Second Military Medical University, 415 Feng Yang Road, Shanghai 200003, P.R. China

E-mail: tianxs04@163.com

${ }^{*}$ Contributed equally

Key words: aquaporin 3, invasion, motility, prostate cancer, matrix metalloproteinase-3, extracellular signal-regulated kinase $1 / 2$

\section{Introduction}

Prostate cancer is one of the most common types of cancer in the USA, and has become the second leading cause of cancer-related mortality among males (1). The presence of invasive cells and of metastasis are the primary factors that contribute to the prognosis of patients with prostate cancer (2). Gene expression microarray technology, which can observe the expression of thousands of genes within a single experiment, has been widely used in the study of cancer. Gene expression profiles have shown that numerous molecular pathways are activated persistently in metastatic processes, and multiple genes are significantly altered during tumor progression (3). Thus, identification of the functions of these pathways and the genes involved in them, may provide important information for use in the development of diagnostic tools and therapies for cancer.

The aquaporins (AQPs) are a large family of small membrane transport proteins that transport either water alone, or water together with small solutes, such as glycerol (4). Thirteen homologous members of the AQP family, which have $25-60 \%$ homology in protein sequence, have been identified in mammalian cells. These AQPs may be further classified into two groups. The first group, includes AQP1, 2, 4, 5 and 8, which selectively transport water alone. The second group includes AQP3, 7, 9 and 10, which transport water and small solutes, including glycerol (5). Recent evidence has shown that AQPs are upregulated in a number of tumors, such as cervical and colorectal cancer $(6,7)$. Studies have shown that AQPs exert a significant impact on cancer metastasis and progression. It has been reported that silencing of AQP1 reduces tumor growth and angiogenesis in mice (8). In addition, knockdown of AQP4 was shown to lead to inhibition of cell invasion in human glioma cells (9), whereas overexpression of AQP8 promoted invasion of cervical cancer cells (10). Studies have also demonstrated that the co-expression of AQP3 and AQP5 in esophageal squamous cell carcinoma correlates with aggressive tumor progression and a poor prognosis (11). Furthermore, silencing of AQP3 has been shown to improve the efficacy of cryotherapy in prostate cancer treatment (12). However, the role of AQP3 in the invasion of prostate cancer cells remains unclear. In the 
present study, the expression of AQP3 in prostate cancer cells was screened and validated. In addition, AQP3 expression was silenced by small interfering RNA (siRNA) in order to investigate the involvement of AQP3 in prostate cancer cell motility and invasion and the possible underlying molecular mechanisms.

\section{Materials and methods}

Materials. A rabbit monoclonal antibody against AQP3 (cat no. sc-20811) and a mouse monoclonal antibody against $\beta$-actin (cat no. sc-8432) were obtained from Santa Cruz Biotechnology, Inc. (Dallas, TX, USA). An extracellular signal-regulated kinase 1/2 (ERK1/2) antibody (cat no. 1240S) and a phospho-ERK1/2 antibody (cat no. 1150S) were purchased from Cell Signaling Technology, Inc. (Danvers, MA, USA). U0126, a specific inhibitor of mitogen-activated protein kinase 1/2 (MEK1/2), was obtained from Sigma-Aldrich (St. Louis, MO, USA).

Cell culture. Cell lines were obtained from the Cell Bank of Chinese Academy of Medical Sciences (Beijing, China). The human PrEC prostate epithelial cell line was grown in PrEMB medium (Clonetics-Biowhittaker, Walkersville, MD, USA) supplemented with $10 \%$ fetal bovine serum (FBS; Sigma-Aldrich). Human LNCap, DU-145, PC-3 and 22RV1 prostate cancer cell lines obtained from the Cell Resource Center of Chinese Academy of Medical Sciences (Beijing, China) were grown in RPMI-1640 (Sigma-Aldrich) supplemented with $10 \%$ FBS. All cell lines were cultured in a $\mathrm{CO}_{2}$ incubator with $5 \% \mathrm{CO}_{2}$ at $37^{\circ} \mathrm{C}$.

cDNA microarray. Total RNA was extracted from PrEC, DU-145 and PC-3 cells. Fluorescently-labeled cDNA was obtained using the Illumina TotalPrep RNA Amplification kit (Ambion Life Technologies, Austin, TX, USA). Hybridization reactions were conducted using Human HT-12 v4 BeadChip (Illumina, San Diego, CA, USA), according to the manufacturer's instructions. The BeadChips were imaged using an Illumina BeadArray reader (Illumina), and the raw data were normalized using an averaging algorithm. The cDNA microarray was conducted by Gene Tech Co., Ltd. (Shanghai, China). The expression of gene transcripts in PrEC cells were defined as 1, and the expression of transcripts in the DU-145 and PC-3 cells was subsequently compared with that of the PrEC cells. Variations $>$ two-fold were taken as a significant difference in gene expression between cell lines. Gene ontology analysis and pathway analysis were then conducted in order to further cluster and analyze these differentially expressed genes.

Reverse transcription-quantitative polymerase chain reaction $(R T-q P C R)$. Cells were harvested and total RNA was isolated using TRIzol $^{\circledR}$ reagent (Invitrogen Life Technologies, Carlsbad, CA, USA). RNA (2 $\mu \mathrm{g})$ was reverse-transcribed into cDNA using M-MLV Reverse Transcriptase (Promega Corporation, Madison, WI, USA). qPCR was then conducted on the cDNA under the following conditions: $95^{\circ} \mathrm{C}$ for $5 \mathrm{~min} ; 95^{\circ} \mathrm{C}$ for $15 \mathrm{sec}$ and $60^{\circ} \mathrm{C}$ for $1 \mathrm{~min}$, for 40 cycles. Primers used in the qPCR were as follows: Forward: CCGTGACCTTTGCCATGTG and reverse: CGAAGTGCCAGATTGCATCATAA for AQP3; forward: AGACCTGGGCAGATTCCAAAC and reverse: CGGCAAGTCTTCCGAGTAGT for MMP-3; and forward: CTGGAACGGTGAAGGTGACA and reverse: AAGGGACTTCCTGTAACAATGCA for $\beta$-actin. The $2^{-\Delta \Delta \mathrm{Ct}}$ method was used to quantify the expression of AQP3 and MMP-3.

Western blot analysis. Cells were washed with ice-cold phosphate-buffered saline (PBS) three times and then lysed in radioimmunoprecipitation assay buffer with protease inhibitor and phosphatase inhibitor cocktails (Roche Applied Science, Mannheim, Germany). The protein concentrations of cell lysates were measured using a Bicinchoninic Protein Assay Reagent kit (Applygen Technologies Inc., Beijing, China). Total protein was boiled with $2 \mathrm{X}$ loading buffer, separated by $10 \%$ SDS-PAGE gel, and transferred to a polyvinylidene difluoride membrane (Invitrogen Life Technologies). The membrane was then blocked with 5\% non-fat milk in Tris-buffered saline with Tween ${ }^{\circledR} 20$ (TBST) for the detection of AQP3, $\beta$-actin and ERK1/2 [or 5\% bovine serum albumin (BSA) in TBST for detection of p-ERK1/2]. The membrane was incubated with the primary antibodies against AQP3, $\beta$-actin, ERK1/2 and p-ERK1/2 overnight at $4^{\circ} \mathrm{C}$. Following $1 \mathrm{~h}$ incubation with the mouse polyclonal secondary antibody (1:3,000; Sigma-Aldrich), the membrane was visualized using an enhanced chemiluminescence detection system (Applygen Technologies Inc.). The densitometry of each band was quantified with Quantity One 4.0 software (Bio-Rad Laboratories, Hercules, CA, USA).

Small interfering (si)RNA. The AQP3-specific siRNA was synthesized by Genepharma (Shanghai, China). A scramble siRNA was used as a control. The sequence of the AQP3 SiRNA was CGAUCAAGCUGCCCAUCUU. DU-145 and PC-3 cells were seeded $\left(1.0 \times 10^{3}\right.$ cells $\left./ \mathrm{ml}\right)$ onto a dish or a plate, and incubated at $37^{\circ} \mathrm{C}$ with $5 \% \mathrm{CO}_{2}$ overnight. Cells were then transfected with siRNA using Lipofectamine ${ }^{\circledR} 2000$ (Invitrogen Life Technologies), according to the manufacturer's instructions. The knockdown efficiency was tested $48 \mathrm{~h}$ later.

Wound healing assay. Cells were seeded onto a 6-well plate at a density of $8.0 \times 10^{5}$ cells $/ \mathrm{ml}$. Cells were further incubated for 24 h until they had reached $\sim 90 \%$ confluence. A $200-\mu 1$ filtered tip was used to create an artificial wound on the confluent cell monolayer. Cells were washed with PBS three times and then cultured in fresh medium without FBS. Images were captured at 0 and $24 \mathrm{~h}$ with a Sony CCD-TR56 (Sony Corporation, Tokyo, Japan) under a microscope (BX51, Olympus, Tokyo, Japan). Five random fields were analyzed in each group and all groups were assayed in triplicate.

Invasion assay. An invasion assay was performed using a 24-well Transwell plate (Costar, San Diego, CA, USA). Briefly, cells were trypsinized and suspended in $200 \mu \mathrm{l}$ serum-free medium at a concentration of $1 \times 10^{5}$ cells $/ \mathrm{ml}$. The upper chambers were coated with Matrigel (Sigma-Aldrich). Cells were then layered in the upper chambers, while RPMI-1640 medium containing 30\% FBS was placed in the 

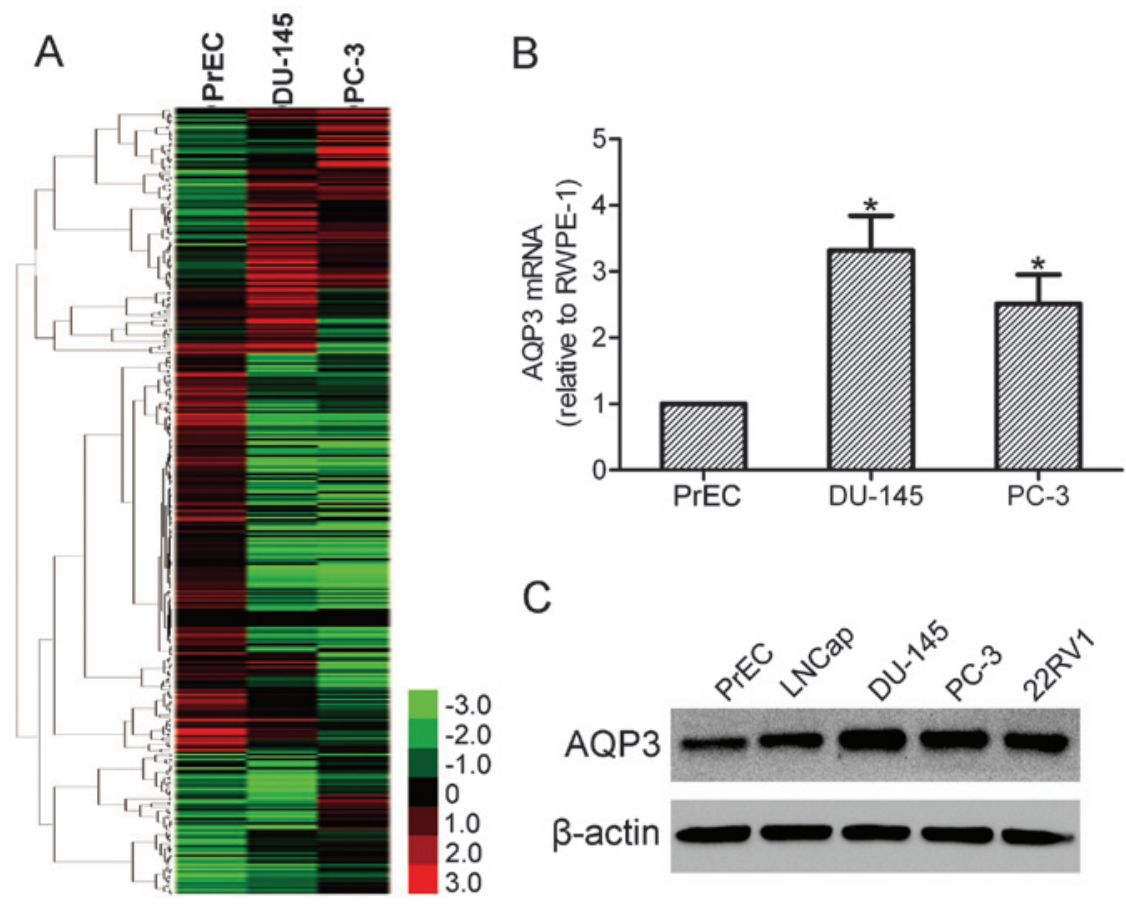

Figure 1. AQP3 expression was elevated in prostate cancer cells. (A) A cDNA microarray was produced, and the genes which were differentially expressed between prostate epithelial cells (PrEC) and prostate cancer cells (DU-145 and PC-3) were clustered. A heatmap was generated from these deferentially expressed genes. (B) RT-qPCR and (C) western blot analysis were conducted to detect the expression of AQP3 in prostate cancer cells. "P<0.05, compared with PrEC cells. AQP3, aquaporin 3; RT-qPCR, reverse transcription-quantitative polymerase chain reaction.

lower chamber. Following incubation in the $\mathrm{CO}_{2}$ incubator for $24 \mathrm{~h}$, cells that had invaded the lower chamber of the wells were fixed with $4 \%$ formaldehyde and stained with crystal violet (Boster Biological Tech Ltd., Wuhan, China). Images from seven visual fields of each well were captured (Sony CCD-TR56) and counted randomly under a light microscope (BX51, Olympus). The mean value for each group was then calculated.

MMP-3 ELISA assay. A matrix metalloproteinase-3 (MMP-3) ELISA assay was performed according to the manufacturer's instructions, in order to assess the level of MMP-3 protein in the culture supernatant. Briefly, following transfection with siRNAs or incubation with U0126 for $24 \mathrm{~h}$, cell supernatant was collected and subjected to the ELISA assay, using an MMP-3 ELISA kit (Calbiochem, Darmstadt, Germany). BSA was used to create a standard curve. Absorbance values were read at $450 \mathrm{~nm}$, and the concentration of MMP-3 was determined by comparing the absorbance values against those of the standard curve.

Statistical analysis. All experiments were repeated three times and data are expressed as the mean \pm standard deviation. Statistical analysis was performed with GraphPad Prism software 5.0 (GraphPad Software, Inc., La Jolla, CA, USA) using Student's t-test or analysis of variance. $\mathrm{P}<0.05$ was considered to indicate a statistically significant difference.

\section{Results}

Overexpression of AQP3 is present in prostate cancer cells. In order to screen the genes that may be involved in the progres- sion of prostate cancer, a cDNA microarray was produced with PrEC, DU-145 and PC-3 cells. Genes expressed in PrEC cells were used as controls. The expression of 685 genes was significantly changed in DU-145 and PC-3 cells compared with PrEC cells (Fig. 1A). Among these genes with significantly altered levels of expression, AQP3 was found to be upregulated in DU-145 and PC-3 cells. In order to validate the cDNA microarray data, qPCR was performed in PrEC, DU-145 and PC-3 cells. The results demonstrated that AQP3 was overexpressed in DU-145 and PC-3 cells compared with PrEC cells (Fig. 1B). Furthermore, increased levels of the AQP3 protein in LNCap, DU-145, PC-3 and 22RV1 cells was also observed by western blot analysis (Fig. 1C). These data indicate that AQP3 may be involved in prostate cancer development and progression.

AQP3 is involved in prostate cancer cell motility. Tumor motility and invasion are essential to the progression and metastasis of prostate cancer (13). Given that AQP3 expression was found to be upregulated in prostate cancer cells, the role of AQP3 in the motility and invasion of prostate cancer cells was further investigated. DU-145 and PC-3 cells were initially transfected with AQP3 siRNA or scramble control siRNA, and the knockdown efficiency was observed using RT-qPCR and western blot analysis (Fig. 2A and B). A wound healing assay was then conducted with AQP3 siRNA-transfected and control cells. The results showed that silencing of AQP3 inhibited the motility of DU-145 and PC-3 cells, indicating an involvement of AQP3 in the regulation of prostate cancer cell motility (Fig. 2C and D).

AQP3 contributes to the invasion of prostate cancer cells. The effect of AQP3 on prostate cancer cell invasion was further 
A

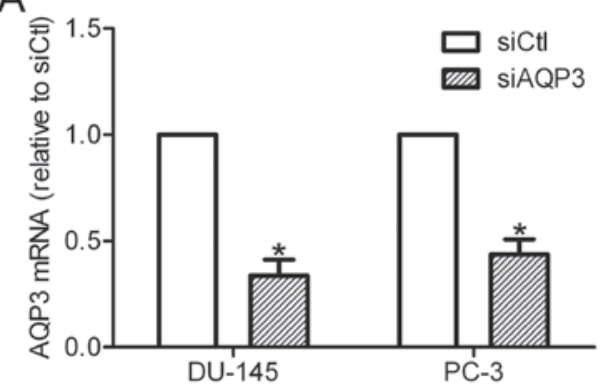

C

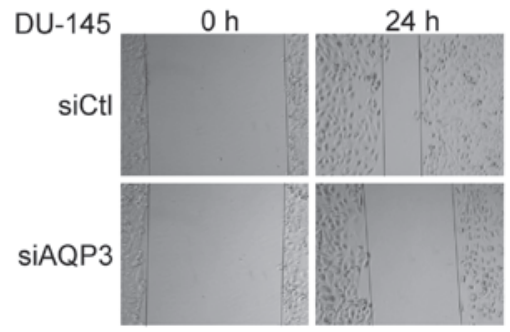

D



B
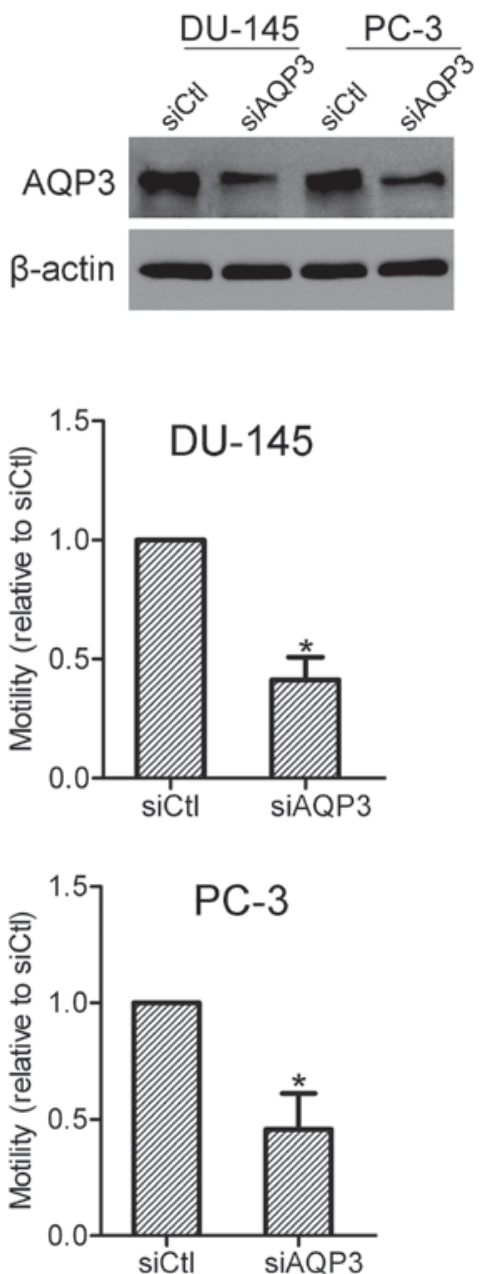

Figure 2. Silencing of AQP3 inhibited the motility of prostate cancer cells. Cells were transiently transfected with siAQP3 or siCtl for $48 \mathrm{~h}$. (A) RT-qPCR and (B) western blotting were performed to detect AQP3 expression in siAQP3 cells and siCtl cells. (C and D) Confluent cell monolayers were wounded with a pipette tip. Representative images were captured at $0 \mathrm{~h}$ and $24 \mathrm{~h}$. The wound closure was quantified and normalized to that of control cells. " $\mathrm{P}<0.05$, compared with siCtl. AQP3, aquaporin 3; RT-qPCR, reverse transcription-quantitative polymerase chain reaction; siAQP3, cells transfected with AQP3-specific small interfering RNA; siCtl, cells transfected with control siRNA.

investigated using an invasion assay. The results showed that AQP3 siRNA-transfected cells exhibited lower invasion capabilities compared with the control cells. This suggests that AQP3 expression affects the invasiveness of prostate cancer cells (Fig. 3).

ERK pathway is required for AQP3-mediated motility and invasion. In order to explore which pathways may be involved in AQP3-mediated motility and invasion of prostate cancer cells, the activation of ERK1/2 in DU-145 and PC-3 cells was detected. As shown in Fig. 4A, the phosphorylation of ERK1/2 was markedly suppressed in AQP3 siRNA-transfected cells compared with that in control cells, suggesting that AQP3 is involved in the activation of the ERK pathway in prostate cancer cells. The function of the ERK pathway in AQP3-mediated motility and invasion was also investigated. A MEK1/2 inhibitor, U0126 $(20 \mu \mathrm{M})$, was used to specifically suppress the activation of the ERK pathway. Notably, U0126 treatment was shown to inhibit the motility and invasion of DU-145 and PC-3 cells (Fig. 4B and C), supporting the hypothesis that the ERK pathway is required for AQP3-mediated motility and invasion.
AQP3 upregulates the expression and secretion of MMP-3 via the ERK pathway. MMP-3 is an important member of the MMP family that is secreted by cancer cells and is known to be involved in invasion and metastasis of cancer cells (14). Therefore, the expression and secretion of MMP-3 was compared between control cells and AQP3 siRNA-transfected cells. RT-qPCR analysis showed that silencing of AQP3 downregulated the expression of MMP-3 mRNA in DU-145 and PC-3 cells (Fig. 5A). In accordance with this finding, the ELISA assay demonstrated that MMP-3 secretion was reduced in AQP3 siRNA-transfected cells compared with control cells (Fig. 5B). These data suggest that AQP3 regulates the expression and secretion of MMP-3 in prostate cancer cells. The role of the ERK pathway in AQP3-mediated MMP-3 expression was further investigated through inhibition of the ERK pathway by U0126. The results showed that U0126 treatment decreased the expression and secretion of MMP-3 in prostate cancer cells (Fig. 5C and D). As AQP3 regulates the ERK pathway and MMP-3 secretion in DU-145 and PC-3 cells, the results suggest that AQP3 increases MMP-3 expression and secretion of prostate cancer cells via regulation of the ERK pathway. 

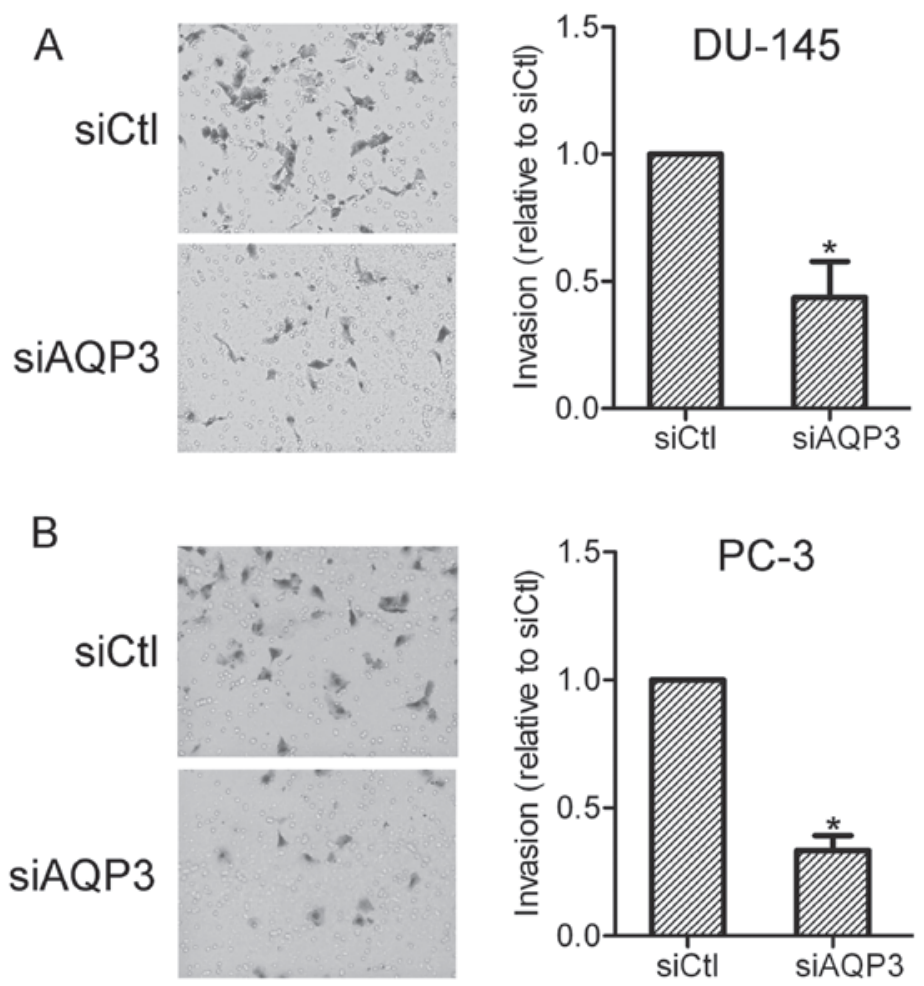

Figure 3. Silencing of AQP3 suppressed the invasion of prostate cancer cells. (A and B) Following transfection with siAQP3 or siCtl, cells were allowed to invade the Transwell plate for $24 \mathrm{~h}$. Results obtained from seven random fields showed that siAQP3 cells exhibited reduced invasion abilities compared with siCtl cells. "P<0.05, compared with siCtl. AQP3, aquaporin 3; siAQP3, cells transfected with AQP3-specific small interfering RNA; siCtl, cells transfected with control siRNA.
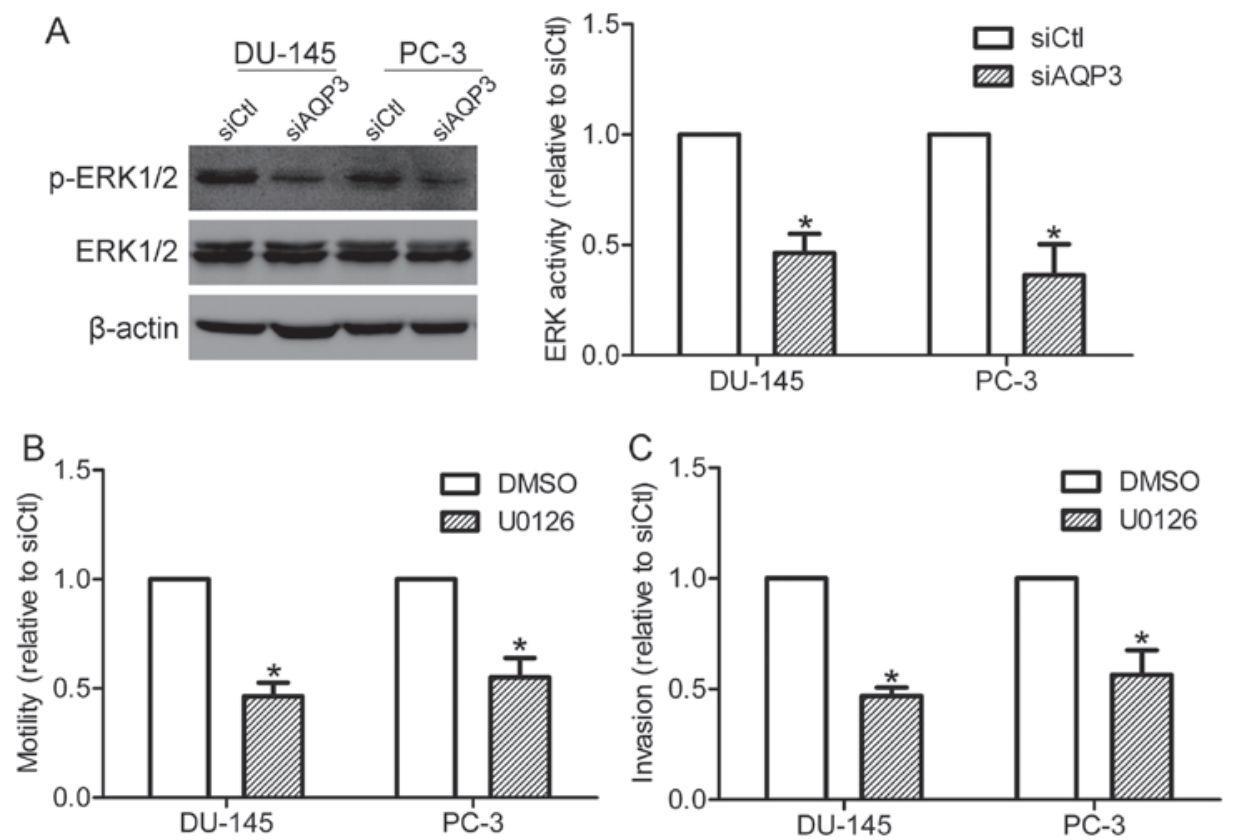

Figure 4. ERK pathway was involved in AQP3-mediated motility and invasion. (A) Cells were tranfected with siAQP3 or siCtl. After $48 \mathrm{~h}$, western blotting was performed to detect the phosphorylation of ERK1/2. DU-145 and PC-3 cells were treated with U0126 (20 $\mu \mathrm{M})$ or DMSO. Then (B) a wound healing assay and $(\mathrm{C})$ an invasion assay were performed to compare the motility and invasion abilities between the DMSO group and the U0126 group. ${ }^{*} \mathrm{P}<0.05$, compared with siCtl. ERK, extracellular signal-regulated kinase; AQP3, aquaporin 3; siAQP3, cells transfected with AQP3-specific small interfering RNA; siCtl, cells transfected with control siRNA; DMSO, dimethyl sulfoxide.

\section{Discussion}

The AQP family is composed of 13 homologous members in mammalian cells. AQP3 has been shown to be upregulated in human gastric carcinoma (15). In the present study, AQP3 was shown to be overexpressed in prostate cancer cells, which 

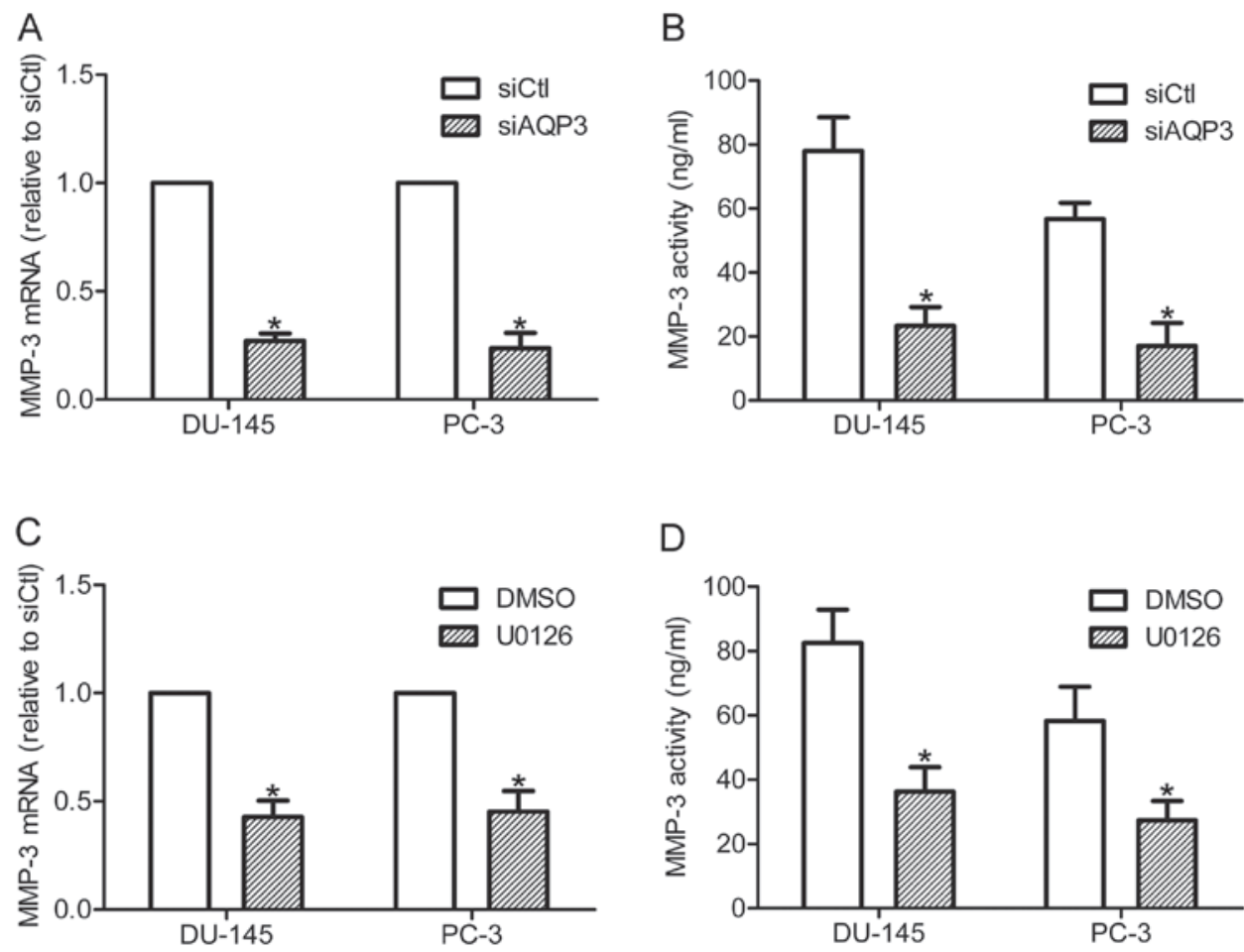

Figure 5. AQP3 increased MMP-3 expression and secretion by activating the ERK pathway. DU-145 and PC-3 cells were transfected with siAQP3 or siCtl, and further incubated in the $\mathrm{CO}_{2}$ incubator for $48 \mathrm{~h}$. (A) MMP-3 mRNA expression was examined by RT-qPCR. (B) MMP-3 secretion was detected by an ELISA assay. DU-145 and PC-3 cells were incubated with U0126 (20 $\mu \mathrm{M})$ or DMSO for $12 \mathrm{~h}$, MMP-3 expression and secretion were examined by (C) RT-qPCR and (D) ELISA assay. "P<0.05, compared with control. AQP3, aquaporin 3; MMP-3, matrix metalloproteinase; ERK, extracellular signal-regulated kinase; siAQP3, cells transfected with AQP3-specific small interfering RNA; siCtl, cells transfected with control siRNA; RT-qPCR, reverse transcription-quantitative polymerase chain reaction; DMSO, dimethyl sulfoxide.

indicates an involvement of AQP3 in prostate cancer progression. Previous studies have shown that AQP3 is required for EGF-enhanced pancreatic cancer cell migration (16), and is important for cell proliferation in esophageal and oral squamous cell carcinoma (17). Clinical studies have reported that AQP3 expression is associated with lymph node metastasis in gastric and colorectal carcinoma $(15,18)$. However, little is currently known regarding the function of AQP3 in prostate cancer. The present study demonstrated that AQP3 may regulate the motility and invasion of prostate cancer cells, further confirming the association between AQP3 and a number of types of malignancy.

AQP3 has been implicated in the regulation of numerous signaling pathways. It has been reported that $\mathrm{AQP} 3$ induces the activation of the phosphoinositide 3-kinase/protein kinase B signaling pathway in human gastric carcinoma cells (19). Knockdown of AQP3 markedly suppresses the p38 MAPK pathway in keratinocytes (20). In the current study, AQP3 was shown to promote the activation of ERK1/2 in DU-145 and PC-3 prostate cancer cells. The ERK signaling pathway, which has been widely investigated in a number of cancer types, contributes to regulation of diverse cellular processes, such as proliferation, survival, differentiation, invasion and metastasis (21). The present study showed that blocking ERK1/2 activation attenuated prostate cancer cell motility and invasion, suggesting a possible role for the ERK pathway in AQP3-mediated motility and invasion.

It is now well-established that the MMP family of proteins enhance tumor invasion and metastasis via degradation of

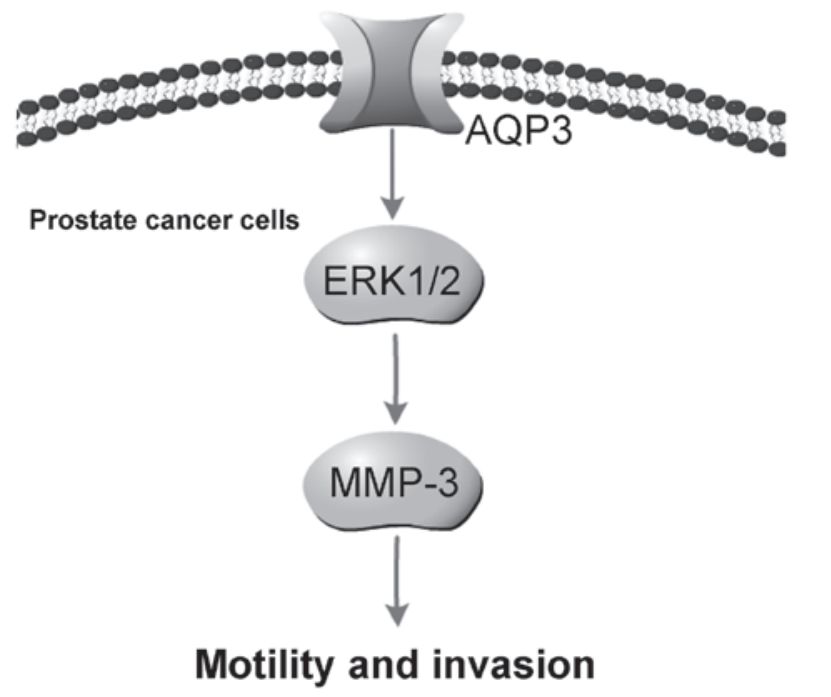

Figure 6. Scheme for the possible pathway of AQP3 in the regulation of prostate cancer cell motility and invasion. AQP3, aquaporin 3; ERK 1/2; extracellular signal-regulated kinase; MMP-3, matrix metalloproteinase-3.

the extracellular matrix (22). As the prominent member of the MMP family, MMP-3 expression has been reported to be associated with metastasis and a poor prognosis in a number of tumors, including prostate cancer $(23,24)$. Studies have shown that AQP3 positively regulates MMP-9 expression in SGC7901 human gastric carcinoma cells (19). The present study demonstrated that AQP3 upregulates the expression 
and secretion of MMP-3 in prostate cancer cells. The ERK pathway has been reported to be respond to the expression of MMPs in numerous types of cancer cells $(25,26)$. In the present study, the results showed that inhibition of the ERK pathway by U0126 treatment decreased the expression and secretion of MMP-3 in prostate cancer cells, suggesting that the ERK pathway is involved in AQP3-mediated MMP-3 expression and secretion in prostate cancer cells.

In conclusion, the present study demonstrated that AQP3 is upregulated in prostate cancer cells. Knockdown of AQP3 suppressed the motility and invasion of DU-145 and PC-3 prostate cancer cells. Furthermore, AQP3 promoted ERK1/2 activation and increased the expression and secretion of MMP-3 in prostate cancer cells. The ERK pathway and MMP-3 are important in prostate cancer cell invasion and metastasis. Blocking of the ERK pathway decreased MMP-3 expression and secretion, and attenuated AQP3-mediated motility and invasion. Therefore, it is possible that AQP3 promotes prostate cancer cell motility and invasion via regulation of ERK1/2-mediated MMP-3 secretion (Fig. 6). In vivo studies are required in order to further determine the effect of AQP3 on tumor cell metastasis.

\section{References}

1. Siegel R, Naishadham D and Jemal A: Cancer statistics, 2013. CA Cancer J Clin 63: 11-30, 2013.

2. Bubendorf L, Schopfer A, Wagner U, et al: Metastatic patterns of prostate cancer: An autopsy study of 1,589 patients. Hum Pathol 31: 578-583, 2000

3. Wang XY, Hao JW, Zhou RJ, et al: Meta-analysis of gene expression data identifies causal genes for prostate cancer. Asian Pac J Cancer Prev 14: 457-461, 2013.

4. Agre $P$, King LS, Yasui M, et al: Aquaporin water channels-from atomic structure to clinical medicine. J Physiol 542: 3-16, 2002.

5. Hara-Chikuma $M$ and Verkman AS: Prevention of skin tumorigenesis and impairment of epidermal cell proliferation by targeted aquaporin-3 gene disruption. Mol Cell Biol 28: 326-332, 2008.

6. Moon C, Soria JC, Jang SJ, et al: Involvement of aquaporins in colorectal carcinogenesis. Oncogene 22: 6699-6703, 2003.

7. Shi YH, Chen R, Talafu T, Nijiati R and Lalai S: Significance and expression of aquaporin 1, 3, 8 in cervical carcinoma in Xinjiang Uygur women of China. Asian Pac J Cancer Prev 13: 1971-1975, 2012.

8. HU J and Verkman AS: Increased migration and metastatic potential of tumor cells expressing aquaporin water channels Faseb J 20: 1892-1894, 2006.

9. Ding T, Ma YJ, Li W, et al: Role of aquaporin-4 in the regulation of migration and invasion of human glioma cells. Int J Oncol 38: 1521-1531, 2011.
10. Shi YH, Rehemu N, Ma H, Tuokan T, Chen R and Suzuke L: Increased migration and local invasion potential of SiHa cervical cancer cells expressing Aquaporin 8. Asian Pac J Cancer Prev 14: 1825-1828, 2013

11. Liu SL, Zhang SY, Jiang H, Yang YX and Jiang Y: Co-expression of AQP3 and AQP5 in esophageal squamous cell carcinoma correlates with aggressive tumor progression and poor prognosis. Med Oncol 30, 2013.

12. Ismail M, Bokaee S, Davies J, Harrington KJ and Pandha H: Inhibition of the aquaporin 3 water channel increases the sensitivity of prostate cancer cells to cryotherapy. Br J Cancer 100: 1889-1895, 2009.

13. Gueron G, De Siervi A and Vazquez E: Advanced prostate cancer: reinforcing the strings between inflammation and the metastatic behavior. Prostate Cancer Prostatic Dis 15: 213-221, 2012.

14. Liu HQ, Song S, Wang JH and Zhang SL: Expression of MMP-3 and TIMP-3 in gastric cancer tissue and its clinical significance. Oncology Lett 2: 1319-1322, 2011.

15. Shen L, Zhu ZC, Huang Y, et al: Expression profile of multiple aquaporins in human gastric carcinoma and its clinical significance. Biomed Pharmacother 64: 313-318, 2010.

16. Liu W, Wang K, Gong K, Li X and Luo K: Epidermal growth factor enhances MPC-83 pancreatic cancer cell migration through the upregulation of aquaporin 3. Mol Med Rep 6: 607-610, 2012

17. Kusayama M, Wada K, Nagata M, et al: Critical role of aquaporin 3 on growth of human esophageal and oral squamous cell carcinoma. Cancer Sci 102: 1128-1136, 2011.

18. Li A, Lu D, Zhang Y, et al: Critical role of aquaporin-3 in epidermal growth factor-induced migration of colorectal carcinoma cells and its clinical significance. Oncol Rep 29: 535-540, 2013.

19. Xu H, Xu Y,Zhang WJ, Shen LZ, Yang L and Xu ZK: Aquaporin-3 positively regulates matrix metalloproteinases via PI3K/AKT signal pathway in human gastric carcinoma SGC7901 cells. J Exp Clin Cancer Res 30: 2011.

20. Hara-Chikuma M and Verkman AS: Aquaporin-3 facilitates epidermal cell migration and proliferation during wound healing. J Mol Med (Berl) 86: 221-231, 2008.

21. Kohno $\mathrm{M}$ and Pouyssegur J: Targeting the ERK signaling pathway in cancer therapy. Ann Med 38: 200-211, 2006.

22. Gialeli C, Theocharis AD and Karamanos NK: Roles of matrix metalloproteinases in cancer progression and their pharmacological targeting. Febs J 278: 16-27, 2011.

23. Jung K, Nowak L, Lein M, Priem F, Schnorr D and Loening SA: Matrix metalloproteinases 1 and 3, tissue inhibitor of metalloproteinase- 1 and the complex of metalloproteinase-1/tissue inhibitor in plasma of patients with prostate cancer. Int J Cancer 74: 220-223, 1997

24. Zhang M, Dai C, Zhu H, et al: Cyclophilin A promotes human hepatocellular carcinoma cell metastasis via regulation of MMP3 and MMP9. Mol Cell Biochem 357: 387-395, 2011.

25. Cho SJ, Chae MJ, Shin BK, Kim HK and Kim A: Akt- and MAPK-mediated activation and secretion of MMP-9 into stroma in breast cancer cells upon heregulin treatment. Mol Med Rep 1: 83-88, 2008.

26. Wang Q, Tang H, Yin S and Dong C: Downregulation of microRNA-138 enhances the proliferation, migration and invasion of cholangiocarcinoma cells through the upregulation of RhoC/p-ERK/MMP-2/MMP-9. Oncol Rep 29: 2046-2052, 2013. 